\title{
О программном обеспечении для планирования мероприятий по снижению потерь моцности от несимметрии токов в сетях 0,4 кB
}

\author{
А.Б. Баламетов 1, д.m.н., npoфpeccop, balametov.azniie@gmail.com
}

A.К. Салимова 1, к.т.н., доиент, ученый секретарь, afag_sk@таil.ru

Н.И. Гаджиев ${ }^{2}$, проректор, старший преподаватель кафедры электромеханики, naib.haciyev.sdu@mail.ru

Э.А. Баламетов ${ }^{3}$, к.т.н., старший диспетчер

${ }^{1}$ Азербайджанский научно-исследовательский и проектно-изыскательский институт энергетики, г. Баку, Аз1012, Азербайджан

${ }^{2}$ Сумгаитский государственный университет, г. Сумгаит, АZ5008, Азербайджан

з ООО "Азеришыг", г. Баку, АZ1008, Азербайджан

Рост мощности электроприемников с нелинейным и несимметричным характером нагрузки ухудшает показатели качества электрической энергии в системах электроснабжения. В результате электрооборудование, рассчитанное на работу в электрической системе при определенном уровне характеристик электрической энергии, эксплуатируется в неэффективных режимах. Это приводит к снижению эффективности работы как самих систем электроснабжения, так и потребителей, подключенных к ним. В результате растет интерес к расчетам потерь мощности от несимметрии токов и напряжений в электрических сетях 0,4 кВ и к соответствующему ПО для оценки потерь мощности от несимметрии токов и напряжений в электрических сетях.

Для определения показателей качества электроэнергии в электрических сетях 0,4 кВ проводились измерения с помощью прибора SIMEAS Q. Напряжение в фазах несимметрично и меняется в пределах 211-228 В, в то же время в конце фидера напряжение меняется в пределах от 145 до 228 В. Изменение активной мощности на головном участке фидера за сутки составляет 2-7 кВт, в фазах нагрузки варьируется в пределах 15-50 \% от полной нагрузки, изменение реактивной мощности по фазам находится в пределах 0,8-1,6 кВАр. У потребителей с нелинейным и несимметричным характерами нагрузки, отдаленных от центра питания, уровни напряжения в течение 30 \% времени дня меньше нормы.

Авторы разработали программу для расчета несимметричных режимов и потерь мощности для четырехпроводных электрических сетей 0,38 кВ режимов с нулевым проводом. Проведен расчет потерь мощности для несимметричных режимов четырехпроводной электрической сети с нулевым проводом.

Ключевые слова: программное обеспечение, электрические сети 0,4 кB, качество электроэнергии, несимметричная нагрузка, несимметрия токов, измерения, показатели качества электроэнергии, потери мощьности.

Увеличение количества и установленной мощности электроприемников с нелинейным и несимметричным характером нагрузки на транспорте и в быту, а также развитие технологических установок в промышленности приводят к ухудшению качества электрической энергии (ЭЭ) в системах электроснабжения. В результате снижается эффективность работы как самих систем электроснабжения, так и потребителей, подключенных к ним. Как следствие электрооборудование, рассчитанное на работу в электрической системе при определенном уровне характеристик ЭЭ, во многих случаях эксплуатируется в неэффективных режимах, что приводит к отрицательным последствиям: увеличение потерь в элементах системы, со- кращение срока службы электрооборудования, ложные срабатывания релейной защиты и автоматики, сбои в работе электронных устройств управления и вычислительной техники, помехи в линиях связи, нарушение нормальной работы производства [1-4].

Несимметрия токов и напряжений влечет за собой увеличение потерь ЭЭ в сетях и элементах ее распределения.

Ущерб от снижения качества ЭЭ обусловлен ухудшением энергетических показателей, снижением надежности функционирования, увеличением потерь активной мощности и потребления активной и реактивной мощностей.

В сетях промышленных предприятий имеет место значительное количество несимметрич- 
ных и нелинейных нагрузок, чувствительных к искажениям напряжений. При снижении качества напряжения ухудшаются условия работы электрооборудования, что приводит к увеличению потерь активной мощности.

На современном этапе развития энергетики достаточно остро стоит вопрос перехода к энергосберегающим технологиям. Это вызвано возрастающим электропотреблением и снижением качества ЭЭ для развивающихся сельскохозяйственных потребителей.

Целью данной работы является анализ путей снижения потерь и повышения качества ЭЭ.

Особенность электрических сетей сельскохозяйственного назначения заключается в большой рассредоточенности потребителей ЭЭ, значительной протяженности сетей 10(6) и 0,4 кВ, в малых величинах потребления в каждом узле [5-8].

В распределительных электрических сетях несимметричной нагрузки с коммунально-бытовыми нагрузками (КБН) применяются трансформаторы со схемой соединения обмоток «звезда-звезда с нулем», обладающие большим сопротивлением нулевой последовательности, примерно на порядок превышающим сопротивление прямой последовательности. При несимметричной нагрузке фаз в этих трансформаторах возникает значительное напряжение нулевой последовательности, вызывающее несимметрию напряжений во вторичных обмотках трансформатора. Коэффициент несимметрии напряжений по нулевой последовательности трансформаторов часто превышает допустимое ГОСТ 13109-97 значение [1, 2].

Данная работа посвящена исследованиям показателей качества ЭЭ (ПКЭ) в сетях 0,4 кВ с КБН. Для измерения ПКЭ в узлах низкого напряжения трансформаторных подстанций 10/0,4 кВ использовались приборы SIMEAS Q.

\section{Результаты экспериментальных исследований ПКЭ в распределительной электрической сети 0,4 кB}

Для определения ПКЭ в электрических сетях 0,4 кВ проводились измерения и испытания на стороне 0,4 кВ трансформаторного пункта. Измерения осуществлялись в летнее время на головной части трехфазного фидера и в точке соединения самого удаленного однофазного абонента с помощью прибора SIMEAS Q. Peзультаты проведенных исследований показали необходимость перехода на новую энергосбе- регающую систему электроснабжения, позволяющую повысить показатели качества ЭЭ.

Графики результатов измерений качества ЭЭ представлены на рисунках 1 и 2.

Графические изменения напряжений в фазах в головном участке фидера показывают, что напряжение летом находится на уровне 210-228 В (рис. 2), то есть на нормальном уровне.

Напряжение в фазах несимметрично и меняется в пределах 211-228 В, то есть 17 В за 18 часов. В то же время на конце фидера напряжение меняется в пределах от 145 до 228 В.

Изменение активной мощности в головном участке фидера за сутки составляет 2-7 кВт.

Результаты экспериментов показывают, что в фазах нагрузка варьируется в пределах

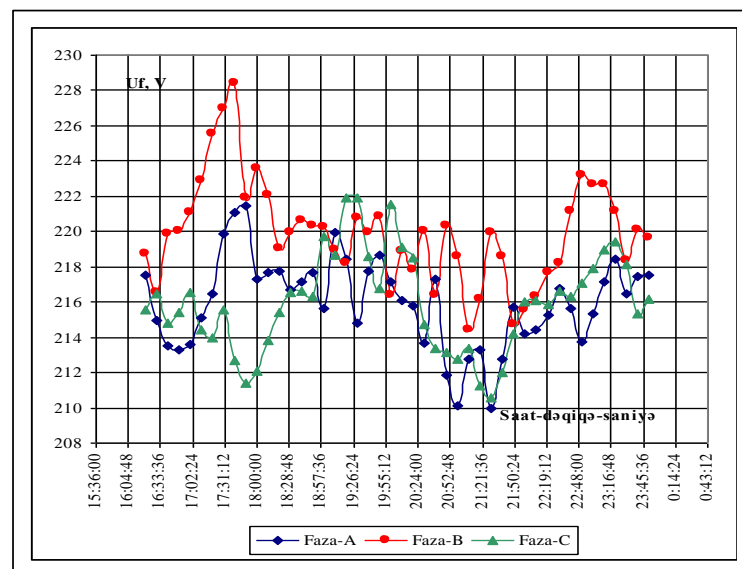

Рис. 1. Графики изменения напряжения в головном участке фидера 0,4 кB

Fig. 1. The voltage change graphs in the head section of the feeder $0,4 \mathrm{kV}$

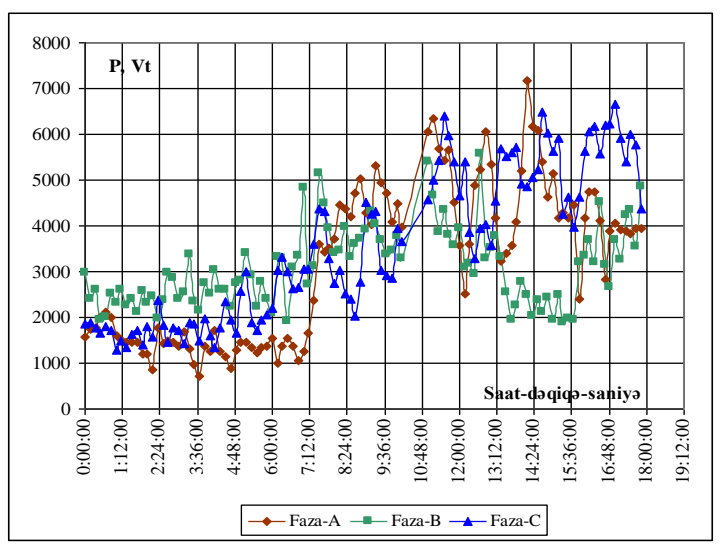

Рис. 2. Графики изменения активной мощңности фазы активной мощности в головном участке

Fig. 2. The active power change graphs in the head section phase 
15,35 и $50 \%$ от трехфазной полной нагрузки. В головном участке фидера изменение реактивной мощности по фазам лежит в пределах $0,8-1,6$ кВАр.

У потребителей несимметричной нагрузки, отдаленных от центра питания на 600-950 м, уровень напряжения в течение $40 \%$ времени суток составляет $5 \%$, для $25 \%$ времени за сутки в норме и в течение $30 \%$ времени дня меньше нормы.

Результаты измерений показали, что токи в отдельных фазах фидеров существенно различаются и изменяются в течение суток, то есть режимы работы сетей несимметричны. Коэффициенты несимметрии токов по обратной и нулевой последовательностям составляют 20-25\%. Коэффициент несимметрии напряжений по нулевой последовательности в часы вечернего максимума нагрузок достигает 7,3\%, что существенно превышает допустимое значение.

При коэффициентах несимметрии токов обратной и нулевой последовательности в сети, равных 0,25-0,30, потери мощности и ЭЭ в линиях и трансформаторах возрастают на $30-50 \%$ по сравнению с симметричным режимом работы [6-9].

Технологические потери ЭЭ в сельских сетях за последнее десятилетие выросли почти в 3 раза и достигают $30 \%[9,10]$. Причиной этого является рост в сетях 0,4 кВ доли однофазной нагрузки по сравнению с трехфазной симметричной нагрузкой. Этому способствовало, в частности, применение мощных (до 50 кВт и выше) однофазных стабилизаторов напряжения.

Существенного снижения потерь ЭЭ в сетях 0,4 кВ можно добиться за счет уменьшения несимметрии токов в этих сетях.

При несимметричной системе токов на участке сети потери мощности $\Delta P_{n s}$ складываются из потерь, обусловленных токами прямой $I_{1}$, обратной $I_{2}$ и нулевой $I_{0}$ последовательностей $[9,10]$ :

$\Delta P_{n s}=\Delta P_{1}+\Delta P_{2}+\Delta P_{0}=3 I_{1}^{2} R_{1}+3 I_{2}^{2} R_{2}+3 I_{0}^{2} R_{0}$,

где $R_{1}, R_{2}, R_{0}$ - активное сопротивление прямой, обратной и нулевой последовательностей участка сети соответственно.

Для расчета потерь мощности на участке сети определим коэффициент потерь мощности от несимметрии токов $K_{n s}$ как отношение потерь мощности при несимметричном режиме работы сети $\Delta P_{n s}$ к потерям мощности при симметричном режиме, обусловленных токами прямой последовательности:

$$
\begin{aligned}
& K_{n s}=\frac{\Delta P_{n s}}{\Delta P_{1}}=1+\frac{I_{2}^{2} R_{2}}{I_{1}^{2} R_{1}}+\frac{I_{0}^{2} R_{0}}{I_{1}^{2} R_{1}}= \\
& =1-K_{2 i}^{2} \frac{R_{2}}{R_{1}}+K_{0 i}^{2} \frac{R_{0}}{R_{1}},
\end{aligned}
$$

где $K_{2 i}=\frac{I_{2}}{I_{1}}, K_{0 i}=\frac{I_{0}}{I_{1}}-$ коэффициенты обратной и нулевой последовательностей токов.

Для трансформаторов и линий электропередачи имеет место $R_{2}=R_{1}$. В таком случае выражение (2) запишется в следующем виде:

$$
K_{n s}=1+K_{2 i}^{2}+K_{0 i}^{2} \frac{R_{0}}{R_{1}} .
$$

Для трехфазной линии с нулевым проводом $R_{0}=R_{\phi}+3 R_{\mathrm{H}}$, поэтому

$$
K_{n s 0}=1+K_{2 i}^{2}+K_{0 i}^{2}\left(1+\frac{3 R_{\mathrm{H}}}{R_{\phi}}\right),
$$

где $R_{\phi}, R_{\mathrm{H}}$ - активное сопротивление фазного и нулевого проводов.

Из выражения (3) видно, что коэффициент потерь мощности от несимметрии токов зависит от коэффициентов несимметрии токов обратной и нулевой последовательностей и от отношения активных сопротивлений нулевой и прямой последовательностей.

Потери ЭЭ в линии напряжением 0,4 кВ обычно в соответствии с ГОСТ 13109-97 определяют по формуле

$$
\Delta W_{\text {н }}=0,7 K_{\text {нер. }} \Delta U_{1} \frac{\tau}{T_{\text {макс }}},
$$

где $\Delta U_{1}$ - потери напряжения в максимум нагрузки сети от шин трансформаторного пункта до наиболее удаленного электроприемника, \%; $K_{\text {нер. }}-$ коэффициент, учитывающий неравномерность распределения нагрузок по фазам.

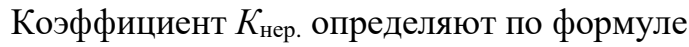

$$
K_{\text {нер. }}=3 \frac{I_{A}^{2}+I_{B}^{2}+I_{C}^{2}}{\left(I_{A}+I_{B}+I_{C}\right)^{2}}\left(1+1,5 \frac{R_{\mathrm{H}}}{R_{\phi}}\right)-1,5 \frac{R_{\mathrm{H}}}{R_{\phi}}
$$

где $I_{A}, I_{B}, I_{C}$ - измеренные токовые нагрузки фаз; $R_{\mathrm{H}} / R_{\Phi}$ - отношение сопротивлений нулевого и фазного проводов.

При отсутствии данных о токовых нагрузках для линий с $R_{\mathrm{H}} / R_{\phi}=1$ следует принимать $K_{\text {нер. }}=1,13$, для линий с $R_{\text {H }} / R_{\phi}=2-K_{\text {нер. }}=1,2$.

\section{Расчет несимметричных режимов четырехпроводной электрической сети с нулевым проводом}

Разработана программа в среде DELPHI для расчета несимметричных режимов и потерь 
мощности для четырехпроводных электрических сетей 0,38 кВ режимов с нулевым проводом [11], блок-схема которой приведена на рисунке 3.

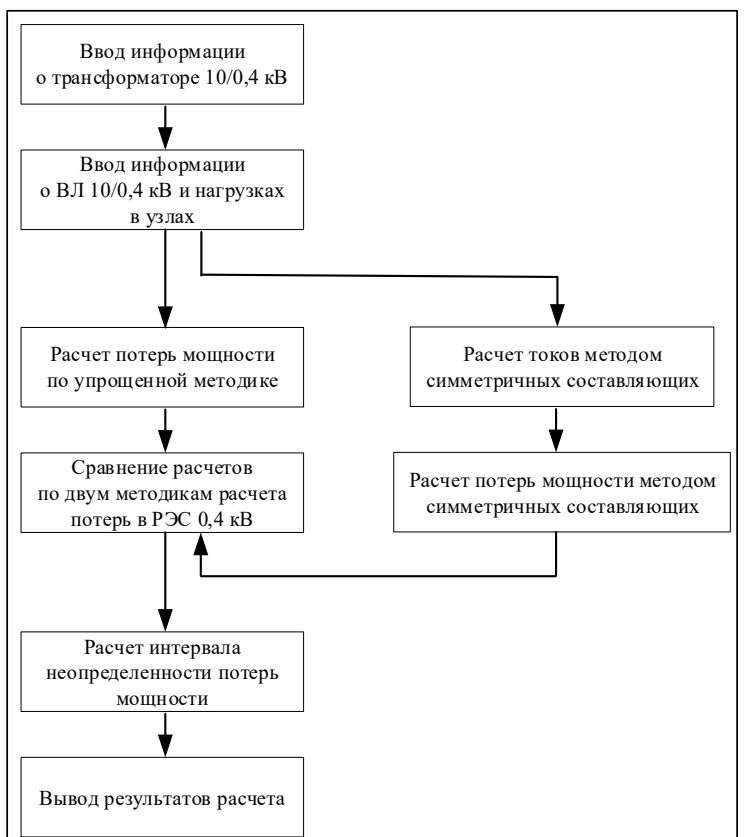

Рис. 3. Блок-схема расчета потерь ЭЭ от несимметрии фидера $0,4 \kappa B$

Fig. 3. Flowchart calculating power losses due to asymmetry 0,4 $\mathrm{kV}$ feeder

Известны методы расчета показателей несимметрии токов и напряжений и коэффициента потерь мощности от несимметрии токов $[10,11]$. В этой программе в качестве критерия потерь применен коэффициент потерь мощности от несимметрии токов. Программа включает следующие этапы:

- расчет потерь мощности в каждой линии и в трансформаторе при симметричной нагрузке;
- расчет потерь мощности, обусловленных несимметрией токов на каждом участке линий и в трансформаторе;

- расчет потерь ЭЭ при несимметричной системе токов в каждой линии, отходящей от данного трансформатора, и в трансформаторе.

Отличительной особенностью программы является ее комплексный характер: одновременно с расчетом потерь ЭЭ в узле сети 0,4 кВ рассчитываются показатели несимметрии напряжений и токов во всех линиях, отходящих от данного трансформатора.

Для моделирования токов методом симметричных составляющих использована стандартная программа [12] (см. http://www.swsys.ru/uploaded/image/2020-2/2020-2-dop/1.jpg).

Пример 1. Расчет для схемы «трансформатор-воздушная линия». Сечение линии 0,38 кВ составляет $25 \mathrm{Mм}^{2}$, удельное сопротивление 1,2 Ом/км, длина - 0,2 км. Сечение нулевого провода - $35 \mathrm{Mм}^{2}$, удельное сопротивление 0,87 Ом/км. Потери холостого хода трансформатора -135 Вт.

Расчеты были проведены для несимметричных одно-, двух- и трехфазных нерегулярных режимов нагрузки электрической цепи (рис. 4).

Сопротивления трансформатора 25 кВА приведены к напряжению 0,4 кВ (табл. 1).

Сопротивления элементов сети, Ом

Таблииа 1

Table 1

Network element resistance, 0 m

\begin{tabular}{|l|c|c|c|c|c|c|}
\hline \multirow{2}{*}{$\begin{array}{c}\text { Элемент } \\
\text { сети }\end{array}$} & \multicolumn{6}{|c|}{ Параметр } \\
\cline { 2 - 7 } & $\boldsymbol{u}_{\boldsymbol{k}}$ & $\boldsymbol{r}_{\mathbf{1}}$ & $\boldsymbol{x}_{\mathbf{1}}$ & $\boldsymbol{z}_{\mathbf{1}}$ & $\boldsymbol{r}_{\mathbf{0}}$ & $\boldsymbol{x}_{\mathbf{0}}$ \\
\hline $\begin{array}{l}\text { Трансфор- } \\
\text { матор } Y / Y_{0}\end{array}$ & 4,5 & 0,154 & 0,244 & 0,287 & 1,650 & 1,930 \\
\hline $\begin{array}{l}\text { Трансфор- } \\
\text { матор } Y / Z_{0}\end{array}$ & 4,7 & 0,177 & 0,243 & 0,302 & 0,073 & 0,0354 \\
\hline Линия & - & 0,24 & 0,077 & 0,252 & 0,762 & 0,308 \\
\hline
\end{tabular}

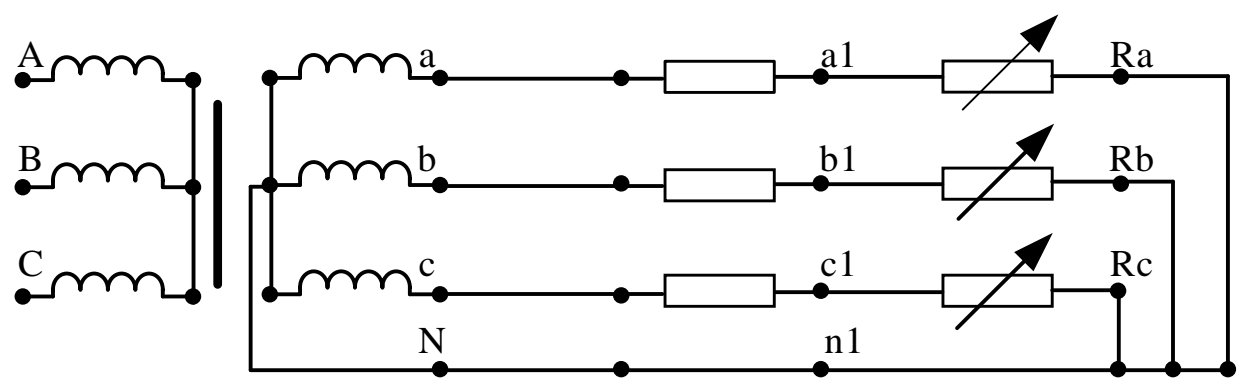

Рис. 4. Расчетная схема распределительной сети 0,4 кB

Fig. 4. The scheme of the distribution network of $0,4 \mathrm{kV}$ 
Сопротивление симметричной нагрузки дано как $Z_{A}=Z_{B}=Z_{C}=7+j 3$ Ом. В несимметричном режиме нагрузка на фазу $A$ в $Z_{A}=7+j 3$ Ом, в других фазах $B$ и $C$ не имеется.

Результаты определения коэффициента потерь мощности от несимметрии токов по несимметричным однофазным режимам нагрузки трансформаторов $Y / Y_{0}$ и $Y / Z_{0}$ сведены в таблицы 2 и 3 соответственно.

Таблиия 2

Результаты расчета в несимметричном режиме

Table 2

Calculation results in asymmetric mode

\begin{tabular}{|c|c|c|c|c|}
\hline \begin{tabular}{|l|} 
№ \\
\end{tabular} & $\begin{array}{l}\text { Элемент } \\
\text { сети }\end{array}$ & $\begin{array}{c}\text { Па- } \\
\text { pa- } \\
\text { метр }\end{array}$ & \begin{tabular}{|c|} 
Еди- \\
ница из- \\
мерения \\
\end{tabular} & $\begin{array}{c}\text { Однофазный } \\
\text { несимметрич- } \\
\text { ный режим }\end{array}$ \\
\hline 1 & \multirow{11}{*}{$\begin{array}{l}\text { Трансфор- } \\
\text { матор } \\
Л Э П ~\end{array}$} & $Z a$ & $\mathrm{OM}$ & $Z_{y}=7+j 3$ \\
\hline 2 & & $Z b$ & $\mathrm{OM}$ & 0 \\
\hline 3 & & $Z c$ & $\mathrm{OM}$ & 0 \\
\hline 4 & & $I_{A}$ & A & 25,82 \\
\hline 5 & & $I_{B}$ & $\mathrm{~A}$ & 0 \\
\hline 6 & & $I_{C}$ & $\mathrm{~A}$ & 0 \\
\hline 7 & & $I_{1}$ & $\mathrm{~A}$ & 9,42 \\
\hline 8 & & $I_{2}$ & A & 9,42 \\
\hline 9 & & $I_{0}$ & A & 9,42 \\
\hline 10 & & $K_{2}$ & o.e. & 1 \\
\hline 11 & & $K_{0}$ & o.e. & 1 \\
\hline 12 & \multirow{5}{*}{$\begin{array}{l}\text { Трансфор- } \\
\text { матор } Y / Z_{0}\end{array}$} & $\Delta P_{1}$ & $\mathrm{BT}$ & 61,21 \\
\hline 13 & & $\Delta P_{2}$ & $\mathrm{BT}$ & 61,21 \\
\hline 14 & & $\Delta P_{0}$ & BT & 20,23 \\
\hline 15 & & $\Delta P_{\varepsilon}$ & $\mathrm{BT}$ & 81,44 \\
\hline 16 & & $K_{\varepsilon}$ & o.e. & 1,33 \\
\hline 17. & \multirow{5}{*}{$\begin{array}{l}\text { ЛЭП 0,38 } \\
\text { кВ }\end{array}$} & $\Delta P_{1}$ & $\mathrm{BT}$ & 63,88 \\
\hline 18 & & $\Delta P_{2}$ & $\mathrm{BT}$ & 63,88 \\
\hline 19 & & $\Delta P_{0}$ & $\mathrm{BT}$ & 202,80 \\
\hline 20 & & $\Delta P_{\varepsilon}$ & BT & 266,68 \\
\hline 21 & & $K_{\varepsilon}$ & o.e. & 4.175 \\
\hline
\end{tabular}

В таблице 3 приведены результаты однофазных несимметричных режимов с трансформатором со схемой соединения $Y / Z_{0}$.

В таблице 4 дается сравнительный анализ результатов расчетов несимметричных режимов.

Результаты исследования показывают, что потери в трансформаторах со схемой соединений $Y / Z_{0}$ меньше, чем в обычных распределительных сетях $Y / Y_{0}$ : при однофазной несимметричной нагрузке в 11 раз, при двухфазной несимметричной нагрузке в 8 раз.

Пример 2. Расчет для схемы «4-проводная воздушная линия». Сечение линии 0,38 кB составляет 25 мм $^{2}$, удельное сопротивление -
1,2 Ом/км, длина - 0,2 км. Сечение нулевого провода -35 мм² $^{2}$.

Результаты расчета по программе в среде DELPHI [11] по упрощенной методике в виде экранной формы приведены на рисунке (см. http://www.swsys.ru/uploaded/image/2020-2/202 0-2-dop/2.jpg).

Таблица 3

Результаты расчета несимметричных режимов с трансформатором $\mathrm{Y} / \mathrm{Z}_{0}$

Table 3

The calculation results for unbalanced modes with a transformer $\mathrm{Y} / \mathrm{Z}_{0}$

\begin{tabular}{|c|c|c|c|c|}
\hline № & $\begin{array}{c}\text { Элемент } \\
\text { сети }\end{array}$ & $\begin{array}{c}\text { Па- } \\
\text { ра- } \\
\text { метр }\end{array}$ & \begin{tabular}{|c|} 
Еди- \\
ница из- \\
мерения
\end{tabular} & $\begin{array}{c}\text { Однофазный } \\
\text { несимметрич- } \\
\text { ный режим }\end{array}$ \\
\hline 1 & \multirow{11}{*}{$\begin{array}{l}\text { Транс- } \\
\text { форма- } \\
\text { тор ЛЭП }\end{array}$} & $Z a$ & OM & $Z_{y}=7+j 3$ \\
\hline 2 & & $Z b$ & $\mathrm{OM}_{\mathrm{M}}$ & 0 \\
\hline 3 & & $Z c$ & $\mathrm{OM}$ & 0 \\
\hline 4 & & $I_{A}$ & A & 20,23 \\
\hline 5 & & $I_{B}$ & A & 0 \\
\hline 6 & & $I_{C}$ & $\mathrm{~A}$ & 0 \\
\hline 7 & & $I_{1}$ & $\mathrm{~A}$ & 9,45 \\
\hline 8 & & $I_{2}$ & A & 9,45 \\
\hline 9 & & $I_{0}$ & A & 9,45 \\
\hline 10 & & $K_{2}$ & o.e. & 1 \\
\hline 11 & & $K_{0}$ & o.e. & 1 \\
\hline 12 & \multirow{5}{*}{$\begin{array}{l}\text { Tранс- } \\
\text { форма- } \\
\text { тор } Y / Y_{0}\end{array}$} & $\Delta P_{1}$ & BT & 54,11 \\
\hline 13 & & $\Delta P_{2}$ & Bт & 54,11 \\
\hline 14 & & $\Delta P_{0}$ & $\mathrm{BT}$ & 728,54 \\
\hline 15 & & $\Delta P_{\varepsilon}$ & $\mathrm{BT}$ & 782,65 \\
\hline 16 & & $K_{\varepsilon}$ & o.e. & 14,46 \\
\hline 17 & \multirow{5}{*}{$\begin{array}{l}\text { ЛЭП } \\
0,38 \text { кВ }\end{array}$} & $\Delta P_{1}$ & $\mathrm{BT}$ & 64,28 \\
\hline 18 & & $\Delta P_{2}$ & $\mathrm{BT}$ & 64,28 \\
\hline 19 & & $\Delta P_{0}$ & $\mathrm{BT}$ & 204,10 \\
\hline 20 & & $\Delta P_{\varepsilon}$ & $\mathrm{BT}$ & 332,67 \\
\hline 21 & & $K_{\varepsilon}$ & o.e. & 4,175 \\
\hline
\end{tabular}

Таблица 4

Результаты расчетов несимметричных режимов

Table 4

The calculation results for asymmetric modes

\begin{tabular}{|c|c|c|c|c|c|c|}
\hline \multirow{2}{*}{$\begin{array}{c}\text { Tранс- } \\
\text { фор- } \\
\text { матор }\end{array}$} & \multicolumn{2}{|c|}{$\begin{array}{c}\text { Одгруз- } \\
\text { ка }\end{array}$} & \multicolumn{2}{|c|}{ Потери } & \multicolumn{3}{c|}{$\begin{array}{c}\text { Нагруз- } \\
\text { ка }\end{array}$} & \multicolumn{2}{|c|}{ Потери } \\
\cline { 2 - 7 } & Вт & Вт & \% & Вт & Вт & \% \\
\hline $\mathrm{Y}^{\mathrm{Y}} \mathrm{Y}_{0}$ & 2865,54 & 1169,42 & 40,81 & 9291,93 & 1166,73 & 12,56 \\
\hline $\mathrm{Y} / \mathrm{Z}_{0}$ & 4666,13 & 473,22 & 10,14 & 10356,44 & 824,87 & 7,96 \\
\hline
\end{tabular}

Из изложенного выше можно сделать следующие выводы: в городских и сельских сетях с КБН уже на стадии проектирования сети необходимо ставить целью снижение потерь и повышение качества ЭЭ [7]. 
Однофазные электроприемники большей мощности нужно включать в трехфазную сеть через симметрирующие устройства.

Проектируемую при симметричном режиме трехфазную сеть с КБН следует рассчитать с помощью программы «Потери энергии», разработанной при несимметричном режиме по статистическим характеристикам несимметрии токов в сетях 0,4 кВ. В зависимости от результатов расчета показателей несимметрии напряжений следует применять трансформаторы $Y / Y_{0}$ или $Y / Z$ н. Если значение расчетного коэффициента $K_{n s}$ менее 1,3 , а коэффициент несимметрии напряжений нулевой последовательности $K_{0 U}$ в $2-3$ раза превышает установленную норму [9, 10, 13-15], следует применять трансформатор $Y / Y_{0}$. Если $K_{n s}$ более 1,3 , а $K_{0 U}$ превышает норму более чем в 3 раза, необходимо применять трансформатор $Y / Z_{0}$.

В условиях эксплуатации сетей с КБН необходимо хотя бы раз в год измерять показатели качества ЭЭ. В тех случаях, когда показатели несимметрии напряжений существенно отличаются от установленных норм, нужно применять следующие меры: отслужившие срок эксплуатации трансформаторы $Y / Y_{0}$ необходимо заменить на трансформаторы $Y / Y_{0}$ или $Y / Z_{0}$; трансформаторы $Y / Y_{0}$ с малым сроком эксплуатации следует временно выводить из работы для проведения ремонта, во время которого устанавливать в нем симметрирующую обмотку (аналогичную трансформатору $Y / Y_{0}$ ); в случаях, когда замена трансформатора $Y / Y_{0}$ невозможна, устанавливать на выходе трансформатора $Y / Y_{0}$ фильтросимметрирующее устройство, которое одновременно со снижением показателей несимметрии напряжений будет компенсировать реактивную мощность [10, 13-15].

Результаты расчета коэффициента несимметрии с учетом разных схем соединения трансформаторов по формулам (2-4) и по общей информации по формуле (6) имеют погрешности в расчете коэффициента несимметрии, доходящие до $50 \%$.

Таким образом, трансформаторы с трансформатором $Y / Z_{0}$ имеют преимущество, заключающееся в наличии несимметричных распределительных сетей напряжением 0,38 кВ.

Применение указанных мер позволит существенно снизить потери и повысить качество ЭЭ в сетях 0,4 кВ с КБН.
Актуальна оценка дополнительных потерь активной мощности, обусловленных отклонением ПКЭ от нормативов.

Значения величин дополнительных потерь мощности в отдельных элементах распределительной сети в условиях несимметричного режима работы необходимы для оценки суммарной доли вышеназванных потерь в общей величине потерь и определения экономического ущерба, обусловленного снижением ПКЭ. Без этих значений затруднительно произвести предварительные расчеты экономической целесообразности проведения мероприятий по повышению качества ЭЭ.

\section{Заключение}

Для определения ПКЭ параметры режима в распределительной электросети 0,4 кВ регистрировались в течение трех дней летнего сезона. Измерения показали, что нормы ПКЭ нарушены и имеется необходимость перехода на современные системы электроснабжения, отвечающие требованиям ПКЭ.

Традиционная схема электроснабжения потребителей на удаленных расстояниях сельских распределительных сетей $10 / 0,4$ кВ зачастую не обеспечивает соответствующее качество ЭЭ. Для этого предлагается переход на схемы электроснабжения, обеспечивающие качество, надежность и эффективность потребителей ЭЭ.

Снижение потерь ЭЭ и повышение ПКЭ с потребителями следует начинать с проектирования сети. Желательно заменять трансформаторы со схемой подключения $Y / Y_{0}$ мощностью 25-100 кВА на $Y / Z_{0}$.

Однофазные потребители большой мощности должны подключаться к трехфазной сети.

Авторы разработали программу для расчета несимметричных режимов и потерь мощности для четырехпроводных электрических сетей 0,38 кВ режимов с нулевым проводом. В электрических сетях низкого напряжения рекомендуется рассчитывать потери ЭЭ с помощью ПО с учетом несимметричности нагрузки.

В результате предложенных мер можно добиться значительного снижения потерь ЭЭ в несимметрично нагруженной электрической сети низкого напряжения и повысить ПКЭ.

\section{תumepamypa}

1. Воротницкий В.Э., Железко Ю.С., Казанцев В.Н., Пекелис В.Г., Файбисович Д.Л. Потери электроэнергии в электрических сетях энергосистем. М.: Энергоатомиздат, 1983. 368 с. 
2. Железко Ю.С., Артемьев А.В., Савченко О.В. Расчет, анализ и нормирование потерь электроэнергии в электрических сетях: Руководство для практических расчетов. М.: НЦ ЭНАС, 2003. 280 с.

3. Железко Ю.С. Потери электроэнергии. Реактивная мощность. Качество электроэнергии: Руководство для практических расчетов. М.: ЭНАС, 2009. 456 с.

4. Железко Ю.С. Методы расчета нагрузочных потерь электроэнергии в радиальных сетях 0,38-20 кВ по обобщенным параметрам схем // Электрические станции. 2006. № 1. С. 31-37.

5. Будзко И.А., Левин М.С. Электроснабжение сельскохозяйственных предприятий и населенных пунктов. М.: Агропромиздат, 1985. 320 с.

6. Левин М.С., Лещинская Т.Б. Анализ несимметричных режимов сельских сетей 0,4 кВ // Электричество. 1999. № 5. С. 18-22.

7. Воротницкий В.Э. Нормирование и снижение потерь электроэнергии в электрических сетях: результаты, проблемы, пути решения // Энергоэксперт. 2007. № 3. С. 10-19.

8. Зотов А.А. Смешанная трехфазно-однофазная система распределения электроэнергии // Энергетик. 2007. № 11. С. 18-22.

9. Косоухов Ф.Д., Васильев Н.В., Филиппов А.О. Снижение потерь от несимметрии токов и повышение качества электрической энергии в сетях 0,38 кВ с коммунально-бытовыми нагрузками // Электротехника. 2014. № 6. С. 8-12.

10. Косоухов Ф.Д., Васильев Н.В., Петров В.Ф., Криштопа Н.Ю. Экспериментальное исследование потерь мощности от несимметрии токов в трехфазных трансформаторах и четырехпроводных линиях 0.38 кВ // Электротехника. 2018. № 2. С. 47-51.

11. Дарахвелидзе П.Г., Марков Е.П. Программирование в Delphi 7. СПб: БХВ-Петербург, 2003. $784 \mathrm{c}$.

12. Littelfuse. Symmetrical component calculator series - relay interface software. URL: https://www. littelfuse.com/products/protection-relays-and-controls/protection-relays/relay-software/symmetrical-componentcalculator.aspx (дата обращения: 30.01.2020).

13. Баламетов А.Б. Методы расчета потерь мощности и энергии в электрических сетях энергосистем. Баку, 2006. 337 с.

14. Баламетов А.Б., Халилов Э.Д. О программе расчета потерь электроэнергии в радиальных электрических сетях // Программные продукты и системы. 2013. № 2. С. 220-224.

15. Balametov A.B., Salimova A.K., Gadzhiev N.I., Balametov E.A. About measures to reduce power losses from current asymmetry in $0.4 \mathrm{kV}$ networks. Proc. 15th ICTPE Conf., Istanbul Rumeli, Turkey, 2019, pp. 17-21.

\section{About software for planning measures to reduce power losses from current asymmetry in $0,4 \mathrm{kV}$ networks}

A.B. Balametov ${ }^{1}$, Dr.Sc. (Engineering), Professor, balametov.azniie@gmail.com

A.K. Salimova ${ }^{1}$, Ph.D. (Engineering), Associate Professor, Scientific Secretary,afag_sk@mail.ru

N.I. Gadzhiev ${ }^{2}$, Vice-rector, Senior Lecturer, Department of Electromechanics, naib.haciyev.sdu@mail.ru

E.A. Balametov ${ }^{3}$, Ph.D. (Engineering), Senior Shift Engineer

${ }^{1}$ Azerbaijan State University of Oil and Industry, Baku, AZ1010, Azerbaijan

${ }^{2}$ Sumgait State University, Sumgait, AZ5008, Azerbaijan

${ }^{3}$ Azerishyg Ltd, Baku, AZ1008, Azerbaijan

Abstract. The increase in the electric receiver power with non-linear and asymmetric load character affects the deterioration of the electric energy quality in power supply systems. As a result, electrical equipment designed to operate in the electrical system at a certain level of electrical energy characteristics is operated in inefficient modes. This leads to a decrease in the efficiency of both the power supply systems themselves and the consumers connected to them. As a result, there is growing interest in calculating power losses from current and voltage asymmetry in $0,4 \mathrm{kV}$ electric networks and in the corresponding software for estimating power losses from current and voltage asymmetry in electric networks.

To determine the electricity quality in $0,4 \mathrm{kV}$ electric networks, measurements were with using the SIMEAS q device. The voltage in the phases is asymmetric and varies between 211-228 V, while at the end 
of the feeder, the voltage varies between 145 and $228 \mathrm{~V}$. The change in active power on the head section of the feeder for a day is $2-7 \mathrm{~kW}$, in the load phases varies within $15-50 \%$ of the full load, the change in reactive power in the phases is within 0,8-1,6 kVAr. Consumers with non-linear and non-symmetrical load patterns that are far from the power center have less than normal voltage levels during $30 \%$ of the day.

The authors developed a program for calculating asymmetric modes and power losses for four-wire electrical networks of $0,38 \mathrm{kV}$ modes with zero wire. There is a power loss calculation for asymmetric modes of a four-wire electric network with zero wire.

Keywords: electric networks $0,4 \mathrm{kV}$, power quality, asymmetric load, current unbalance, measurements, power quality indicators, power losses.

\section{References}

1. Vorotnitsky V.E., Zhelezko Yu.S., Kazantsev V.N., Pekelis V.G., Faybisovich D.L Energy Loss in Electric Networks of Power Systems. Moscow, 1983, 368 p. (in Russ.).

2. Zhelezko Yu.S., Artemev A.V., Savchenko O.V. Calculation, Analysis and Rationing of Energy Losses in Electric Networks: A Practical Guide. Moscow, 2003, 280 p. (in Russ.).

3. Zhelezko Yu.S. Energy Loss. Reactive Power. Power Quality: A Practical Guide. Moscow, 2009, 456 p. (in Russ.).

4. Zhelezko Yu.S. Methods for calculating electricity load losses in radial networks of $0.38-20 \mathrm{kV}$ according to the generalized circuit parameters. Power Plants, 2006, no. 1, pp. 31-37 (in Russ.).

5. Budzko I.A., Levin M.S. Power Supply of Agricultural Enterprises and Settlements. Moscow, Agropromizdat Publ., 1985, 320 p. (in Russ.).

6. Levin M.S., Leshchinskaya T.B. Analysis of asymmetric modes of rural networks 0.4 kV. Electricity, 1999, no. 5, pp. 18-22 (in Russ.).

7. Vorotnitsky V.E. Rationing and reduction of energy losses in electric networks: results, problems, solutions. Energoekspert, 2007, no. 3, pp. 10-19 (in Russ.).

8. Zotov A.A. Mixed three-phase-single-phase power distribution system. Power Engineer, 2007, no. 11, pp. 18-22 (in Russ.).

9. Kosoukhov F.D., Vasilev N.V., Filippov A.O. Reducing losses from current asymmetry and improving the quality of electric energy in networks of $0.38 \mathrm{kV}$ with household loads. Electrical Engineering, 2014, no. 6, pp. 8-12 (in Russ.).

10. Kosouhov F.D., Vasilev N.V., Petrov V.F., Krishtopa N.Yu. An experimental study of power losses due to current asymmetry in three-phase transformers and four-wire $0.38 \mathrm{kV}$ lines. Electrical Engineering, 2018 , no. 2, pp. 47-51 (in Russ.).

11. Darakhvelidae P.G., Markov E.P. Delphi 7 Programming. St. Petersburg, 2003, 784 p. (in Russ.).

12. Littelfuse. Symmetrical Component Calculator Series - Relay Interface Software. Available at: https://www.littelfuse.com/products/protection-relays-and-controls/protection-relays/relay-software/symmetrical-component-calculator.aspx (accessed January 30, 2020).

13. Balametov A.B. Methods for Calculating Power and Energy Losses in Electric Networks of Power Systems. Baku, 2006, 337 p. (in Russ.).

14. Balametov A.B., Halilov E.D. About the program of electric power losses calculation in radial electric mains. Software \& Systems, 2013, no. 2, pp. 220-224 (in Russ.).

15. Balametov A.B., Salimova A.K., Gadzhiev N.I., Balametov E.A. About measures to reduce power losses from current asymmetry in $0.4 \mathrm{kV}$ networks. Proc. 15th ICTPE Conf., Istanbul Rumeli, Turkey, 2019, pp. 17-21.

\section{Для цитирования}

Баламетов А.Б., Салимова А.К., Гаджиев Н.И., Баламетов Э.А. О программном обеспечении дмя планирования мероприятий по снижению потерь мощности от несимметрии токов в сетях 0,4 кB / / Программные продукты и системы. 2020. T. 33. № 2. C. 328-335. DOI: $10.15827 / 0236-$ 235X.130.328-335.

\section{For citation}

Balametov A.B., Salimova A.K., N.I. Gadzhiev, Balametov E.A. About software for planning measures to reduce power losses from current asymmetry in 0,4 kV networks. Software \& Systems, 2020, vol. 33, no. 2, pp. 328-335 (in Russ.). DOI: 10.15827/0236-235X.130.328-335. 